\title{
Evaluation of Chest X-Ray Quality Parameters
}

\section{Dhiego Donizethe Ferreira Gumieri ${ }^{1 *}$ and Israel de Souza Marques ${ }^{2}$}

${ }^{1}$ Teacher at the Department of Medicine/Faculty of Medical Sciences/Imaging, State University of Mato Grosso, Brazil ${ }^{2}$ Student in the Course of Medicine/Faculty of Medical Sciences/Imaging, University of the State of Mato Grosso, Brazil

*Corresponding author: Dhiego Donizethe Ferreira Gumieri, Teacher at the Department of Medicine/Faculty of Medical Sciences/Imaging, State University of Mato Grosso, Brazil

\begin{abstract}
Proper control of radiographic quality is important to guarantee the method high sensitivity and specificity, important factors for diagnostic complementation. It is the responsibility of the practitioner performing the radiological techniques to ensure that all chest radiographs are provided for minimal rotation, good radiographic exposure and adequate inspiration. This cross-sectional study retrospectively evaluated 456 chest radiographs in the poster anterior and lateral projection performed at a diagnostic center between May 2017 and September 2019. 45\% had changes in technical parameters, of which $62 \%$ had positioning errors (rotation and asymmetry between the spinous process and medial extremity of the clavicles), $25 \%$ had errors in radiographic exposure (underexposure and overexposure) and 13\% had inadequate respiratory maneuver. The observed results indicate significant deficiency of professionals regarding the minimum quality control required, as well as the need to implement a permanent education plan about the method.
\end{abstract}

\section{Keywords}

Quality control, Chest X-ray, Technical parameters

\section{Introduction}

Chest X-ray examination is the most requested and observed in outpatient clinics and emergencies. Also examination is most likely to be reviewed exclusively by the radiologist [1]. Chest $\mathrm{X}$-rays is to intended to confirm the information obtained by the history and physical examination [2]. The correct quality control on the technical parameters comprises part of the systematic survey [3]. And it is up to the professional radiological techniques such analysis, prior to dispensing the radiographic examination. It should be employed to analyze and, if necessary correct the rotation parameters, and display patient's inspiration
[4]. In a properly exposed radiograph medial the clavicles ends must be equidistant from the middle line of the patient, indicating the absence of rotation, the thoracic vertebrae must be discernible weakly through the heart area, and the correct maneuver inspiratory characterized expandability pulmonary appropriate by negative contrast, air, favoring the evaluation of lung and mediastinal field changes [5].

This study aims to evaluate the technical parameters of quality of chest radiographs in a radiology unit in the city of Araputanga - Mato Grosso.

\section{Materials and Methods}

This cross-sectional study was conducted at the Radiology Service Araputanga Health Center - MT, between May 2017 and September 2019 were evaluated retrospectively 456 chest $\mathrm{X}$-rays in the posterior-anterior and left lateral considering diagnosis and suitable breathing maneuver.

The tests were carried out in conventional apparatus $500 \mathrm{~mA}$ together to Computed Radiography System RFF - type phosphor radiography photostimulable. A radiographic analysis was based on the observation of the following technical criteria: Positioning, exposure and suitable breathing maneuver (apnea maximum inspiratory) as the following criteria, and subsequently forwarded to the radiologist to prepare the radiographic report.

\section{Positioning}

In the posterior-anterior (PA) comprises positioning appropriate minimum speed and mediastinal vascular pedicle, this factor is regarded as the equidistance 

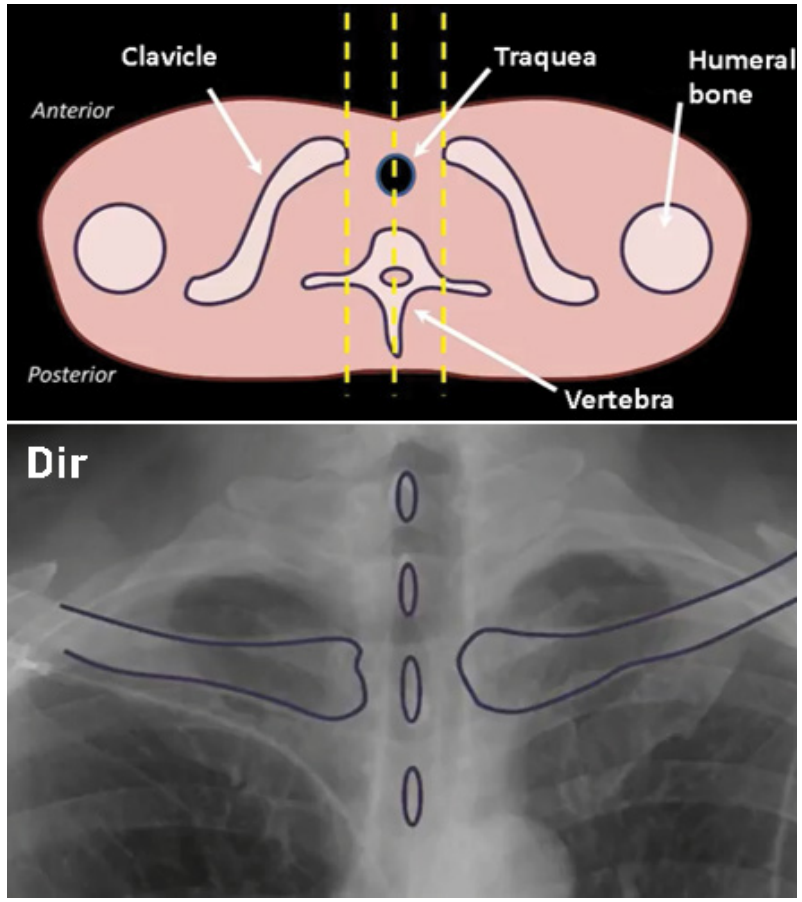

Figure 1: Illustration transversal cutting and radiography showing no rotation symmetry between the spinous process and the end of the clavicles bilaterally.

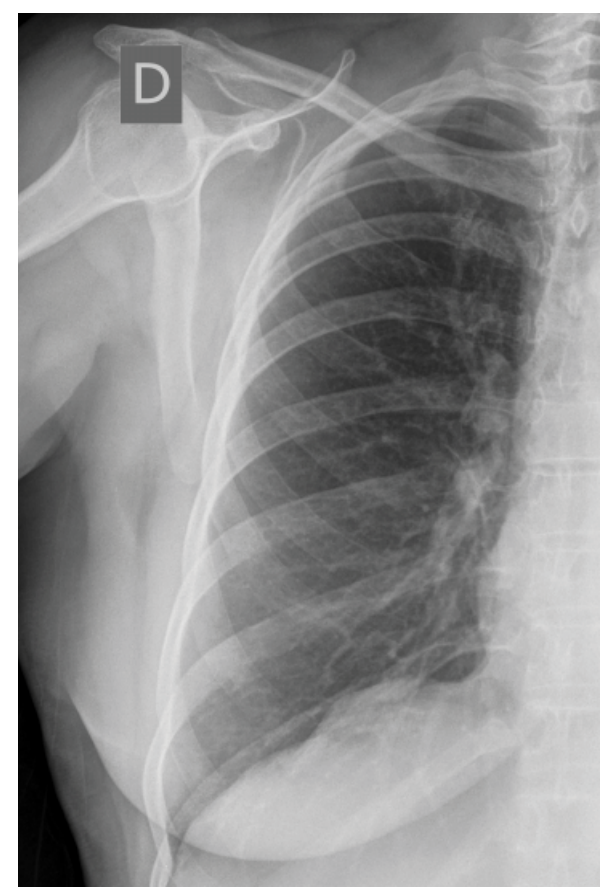

Figure 2: Radiography well positioned, the free lung field showing overlapping of the right scapula.

between the spinous process and the medial edge of the clavicles (Figure 1).

Although the incidence of posterior - anterior (PA), the shoulder blades should be designed laterally to the lung field, for this to be possible, it is necessary to put the hand back in the posterior region of the iliac spines and slightly rotate the shoulders, touching the anterior region the image receptor [6]. The overlap adjacent to the scapula can simulate lung field opacities, pleural

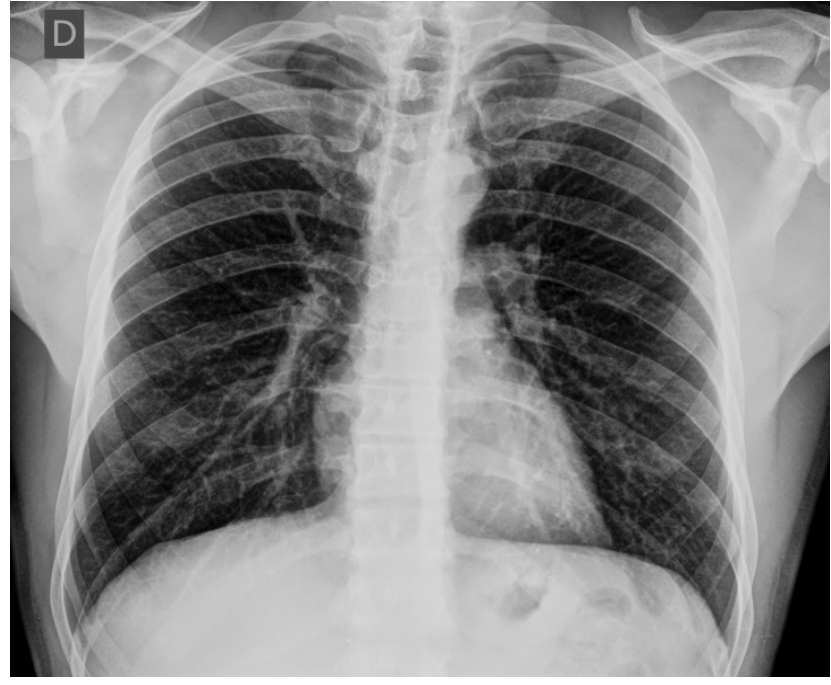

Figure 3: Chest X-ray on the exposed anterior-posterior projection appropriately presenting levels of density and absolute radiographic contrast.

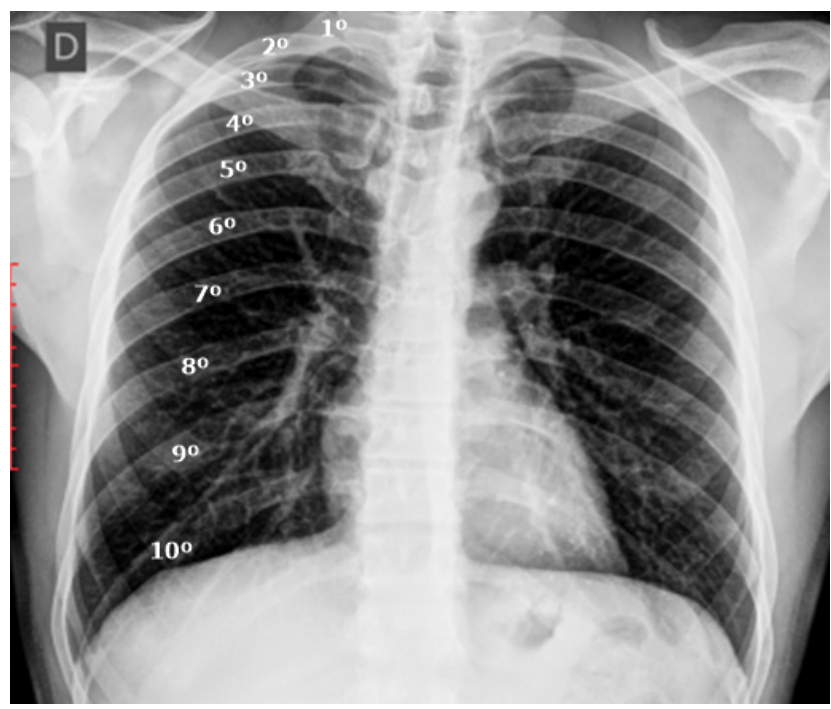

Figure 4: Standard radiography properly inspired, showing ten (10) posterior ribs on the diaphragm.

lines or edges (pneumothorax) (Figure 2).

\section{Radiographic exposure}

Three physical factors determine the quality of radiography, contrast, sharpness and quantum noise. Contrast refers to the density difference between the areas of the radiograph, which allows the display of information contained [7].

The darkening of the film or degree of blackening is a direct response from the number of photons that strike the film, and is usually measured as optical density.

The variation of DO is represented in the film by shades of gray [8].

Pragmatically, it is important to visualize the pulmonary vascular, cardiac and heart retro, bone structures and upper floor of the abdomen (Figure 3). 


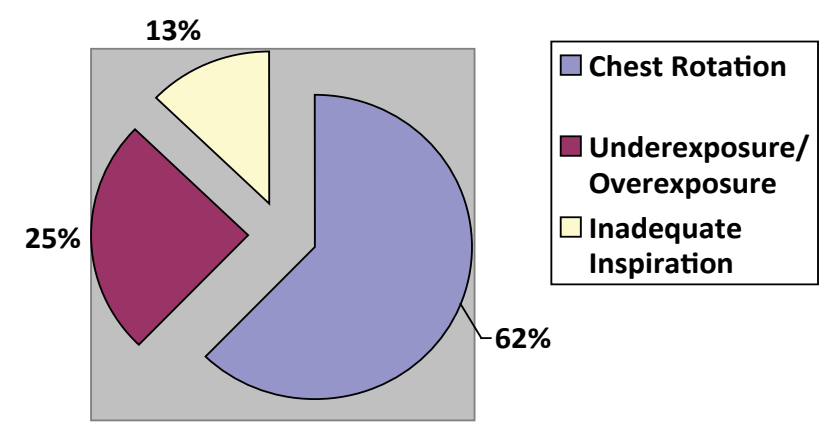

Figure 5: Distribution of frequencies of the quality parameters evaluated in the study.

\section{Maximum inspiratory apnea}

The temporary respiratory paralysis is deliberately important in chest radiographs, because any chest movement or lung occurring during the exposure will result in image blurring [9].

Already maximum inspiration has two objectives: The properly inflated lungs of air contrast negatively contemplating the entire anatomy, and avoid unsatisfactory analysis of the size of the cardiac silhouette, which can apparently appear expanded as lung volume will be less, such a relationship is useful in the measurement of ICT - cardiothoracic index [9].

In practice, a good maximum inspiratory apnea relates to viewing the posterior segment of the rib 9 or 10 on the diaphragm (Figure 4).

\section{Results}

Among the 456 evaluated radiographs, 45\% (n $=205$ ) radiographic changes were identified in the technical parameters. Of these rays $205,62 \%(n=127)$ showed radiographic positioning errors, $25 \%(n=51)$ showed radiographic exposure errors and $13 \%(\mathrm{n}=$ 26) radiographs showed respiratory failure maneuver (Figure 5).

\section{Discussion}

Lacey and colleagues [10], they categorized the speed of the chest and positioning errors that can distort the contours of the vascular pedicle (superior vena cava and aortic vessels of the trunk above may simulate mediastinal mass).

Felson and colleagues [11] underexposure reported as consequences of the difficulty in assessing the lung fields and possible fractures of the ribs, and pulmonary pseudo-hyperinflation in cases of radiographic over exposure.

\section{Conclusions}

Based on the results, the following conclusions were established:

The professional who performs the radiological techniques, be it technical or technologist in radiology to propose an adequate systematization for evaluation of technical parameters and correct them when necessary.

One should avoid as much unnecessary repetition, whose goal aims to reduce the dose used in the realization of new radiation exposure.

The observed results also indicated that the quality control runs through radiological examinations for continuing education of these professionals, an important factor for the realization of a chest X-ray of excellent standard and that further studies should be conducted to clarify its impact on image quality obtained.

\section{Acknowledgment}

Araputanga the Health Center and Health Municipal Secretary Emerson José Prado, and all the workers of the State University of Mato Grosso.

\section{Interest Conflicts}

No conflicts of interest.

\section{References}

1. (2019) University of $E$ de $M G$ Gumieri, "ABC... in the systematic assessment of the chest," Curie \& Roentgen.

2. Capilheira MF (2006) Epidemiology of complementary test ordering in medical consultations population-based study of the epidemiology of diagnostic test. Diabetes 40: 289-297.

3. Daffner R (2013) Clinical radiology - The essentials. (3 edn), USA.

4. WC da Silva, MA Marques, AV do Nascimento (2013) Comparative study for the assessment of technical failures in conventional chest radiographs. Radiol Bras 46: 39-42.

5. Clarke C, Dux A (2011) Chest X-rays for medical students. ( $1^{\text {st }}$ edn), Oxford: Wiley-Blackwell.

6. Bontrager K, Lampignano J (2015) Treatise on positioning and radiographic and associated anatomy. ( $8^{\text {th }}$ edn), Elsevier.

7. Prando, Moreira F (2015) Fundamentals of radiology and diagnostic imaging. ( $2^{\text {nd }}$ edn), São Paulo: Elsevier.

8. Muller I, Muller N (2017) Chest - CBR. ( $2^{\text {nd }}$ edn), Rio de Janeiro: Elsevier.

9. Foltran C, Pizzol LR, de Santana MLGC, Fernandes MR (2015) Assessment of knowledge in the correct ordering of imaging exams for 5th and 6th year inmates at the Faculty of Medicine, University of Mogi das Cruzes, SP. Rev Med 94: 126.

10. Lacey G, Morley S, Berman L (2008) Chest X-ray. Rio de Janeiro: Elsevier.

11. Goodman L (2001) Felson: Principles of chest radiology. $\left(2^{\text {nd }} e d n\right)$, Atheneu. 